\title{
Anestesiología en la Seguridad de Paciente Quirúrgico
}

\author{
JOEL MARCHANT K. ${ }^{1}$, JAVIER WEBAR V., MICHAELA NARDIELLO , GUSTAVO GALLEGUILLOS. ${ }^{2}$
}

Servicio de Anestesia, Hospital Guillermo Grant Benavente. Concepción.

Becarios Anestesiología Universidad de Concepción.

\section{Estado del Arte}

Han transcurrido años, desde que los hospitales eran recintos sobrepoblados y con malas condiciones de salubridad y seguridad, atendidos por personas de congregaciones religiosas que carecían de conocimientos médicos. La atención que recibían los pacientes era de tipo espiritual para morir en presencia de Dios ${ }^{1}$. Los avances de la medicina y tecnología provocaron un impacto en el enfoque de estos recintos, al ofrecer tratamiento para distintos tipos de enfermedades.

Esto permitió un aumento de la expectativa de vida de la población, la aparición y diagnóstico de nuevas patologías en personas de mayor edad, lo cual provocó un significativo aumento tanto en la demanda como en los gastos de los sistemas de salud. A medida que las instituciones de salud comenzaron a realizar tratamientos para diversas enfermedades, cada vez más complejas, el concepto de seguridad del paciente comenzó a ser una preocupación de las entidades encargadas de brindar atención sanataria en diferentes países, con la finalidad de evitar la aparición de complicaciones, hoy llamados eventos adversos, del paciente durante su atención. A nivel mundial se comenzaron a realizar estudios sobre seguridad, con la finalidad de identificar los errores que puede cometer el personal que atiende a un paciente, conocer los factores que desencadenan estos errores y el daño que pueden producir al paciente.

Los estudios de seguridad comenzaron a utilizar Sistemas de Notificación de Incidentes. El primer país a nivel mundial que los utilizó fue
Australia en 1987, siendo Suiza, el primero en utilizar estos sistemas mediante internet, para distribuir los resultados de sus estudios a nivel mundial ${ }^{2}$. La declaración de seguridad de Helsinki creada por la European Board of Anaesthesiology con cooperación de la European Society of Anaesthesiology y aprobada por la OMES, la World Federation of Societies of Anaesthesiologists y la European Patients Federation en el encuentro de anestesiología de Europa realizado en Junio del año $2010^{3}$, estableció que todos los países Europeos que presten servicios de anestesiología deben utilizar estos sistemas.

En Chile existe un estudio de incidentes, realizado el año 2008 en el hospital Padre Alberto Hurtado. Este fue elaborado en base a los proyectos realizados en España, tales como IDEA (2004) y ENEAS (2005), enfocandose en los eventos adversos ligados a la hospitalización en las diferentes áreas de las Unidades de Gestión Clínica (UGC) ${ }^{4}$.

El 13 de abril del 2012 en Chile se promulgo la ley $20.584^{5}$. Dicha ley esta orientada a la regulación de los derechos y deberes de las personas en atención de salud, destacando el artículo 4, el cual menciona "Toda persona tiene derecho a que, en el marco de la atención de salud que se le brinda, los miembros del equipo de salud y los prestadores institucionales cumplan las normas vigentes en el país, y con los protocolos establecidos, en materia de seguridad del paciente y calidad de la atención de salud, referentes a materias tales como infecciones intrahospitalarias, identificación y accidentabilidad de los pacientes, errores en la atención de salud y, en general,

Correspondencia a:

Joel Marchant K.

joelmarchantkemp@gmail.com 
todos aquellos eventos adversos evitables según las prácticas comúnmente aceptadas. Adicionalmente, toda persona o quien la represente tiene derecho a ser informada acerca de la ocurrencia de un evento adverso, independientemente de la magnitud de los daños que aquel haya ocasionado". Durante ese mismo año en el $40^{\circ}$ Congreso Chileno de Anestesiología realizado en Valdivia Chile la Sociedad de Anestesiología de Chile, se adhirió a la declaración de Seguridad de Helsinki. Sin embargo, hasta el día de hoy no existe un sistema de registro computacional que permita realizar el reporte y analisis de incidentes con una taxonomía común para nuestro país.

El Ministerio de Salud estableció un sistema de acreditación que consiste en un proceso de mejoramiento continuo, respecto al cumplimiento de un estándar mínimo, de acuerdo al tipo de establecimiento y a la complejidad de las prestaciones ${ }^{6}$.

La seguridad en salud, tiene como objetivo reducir el riesgo de daño de pacientes, profesionales y familiares asociado a la atención sanitaria, hasta un mínimo aceptable ${ }^{7}$. Este daño es derivado por un incidente, generado por un error o un incumplimiento, que puede ocasionar un daño innecesario al paciente, diferenciándose en cuasi-incidente, incidente sin daño y evento adverso, dependiendo del impacto que generan en el paciente. Los cuasi-incidente corresponden al $75 \%$ de los incidentes registrados y se definen como los incidentes que no alcanzan al paciente, los incidentes sin daño corresponden a los incidentes que alcanzan al paciente y no producen daño, y los eventos adversos son los incidentes que alcanzan la paciente y producen un daño ${ }^{8}$.

Un error es considerado como el hecho de no llevar a cabo una acción prevista o de aplicar un plan incorrecto en la fase de planificación o ejecución, manifestándose como errores de comisión (al realizar algo incorrecto) o como error de omisión (al no hacer lo correcto), a diferencia de la infracción que es el desvió deliberado de las normas, reglas o procedimientos operativos. Los errores pueden producirse por insuficiente preparación técnica, falta de habilidad o por ignorancia de las reglas que rigen una actividad, falla del equipamiento médico, organización y condiciones de trabajo.

El interés por la seguridad en la atención de salud ha aumentado significativamente en los úl- timos 20 años, la realización de distintos estudios permiten estimar la incidencia y la gravedad de los eventos adversos en la atención sanitaria y sus costos asociados. El Instituto de Medicina de los Estados Unidos de Norteamérica estima que se producirá un evento adverso en el 3 a $4 \%$ de los ingresos hospitalarios, pudiendo ocasionar la muerte del paciente en un 7 al 14\%. Estudios realizados en España han determinado que pacientes hospitalizados mayores de 65 años tienen incidencia media de los eventos adversos del $9 \%$, alcanzando el $15 \%$ en otras instituciones ${ }^{9-10}$. Otros estudios a nivel mundial indican que el $50 \%$ de los eventos adversos son evitables, además que el $75 \%$ de los errores cometidos en las instituciones de salud se deben al factor humano y el $30 \%$ de ellos se deben a una violación de la práctica o estándares establecidos ${ }^{11}$.

Se ha podido demostrar en distintos países que los errores humanos tienen una importante contribución a la morbilidad y mortalidad en la medicina. El ser humano no es perfecto, y por lo tanto puede cometer errores, es necesario trabajar para alcanzar un nivel de seguridad de "complicaciones cero", que evite la generación de incidentes y que minimice el daño que puedan generar los incidentes que sean cometidos. Las medidas necesarias para generar estos efectos en la seguridad consisten en elaborar sistemas de procedimientos seguros, lograr cambios culturales con el objetivo de educar u orientar a las personas a ser honestas y reporten cuando haya ocurrido un incidente, sabemos que este punto es difícil en nuestro país, por la judialización de la medicina, pero es un tema que debe ser debatido y aclarado en las instancias correspondientes, se debe evitar el castigo de los errores, generar medidas que eviten los incidentes y finalmente creando un sistema de análisis que facilite su seguimiento.

La cultura de seguridad se define como un patrón integrado de comportamiento individual y de la organización, basado en creencias y valores compartidos, que busca continuamente reducir al mínimo el daño que podría sufrir el paciente como consecuencia de los procesos y prestaciones. La barrera más difícil de generar esta cultura de seguridad es la resistencia al cambio por parte del personal, debido a esto, el cambio cultural puede tomar cierto tiempo en ser generado a nivel global dentro de una institución de salud. 
Para generar un cambio cultural es necesario:

- Educar y formar al personal en materia de seguridad.

- Conocer las actividades de alto riesgo y las más propensas a errores.

- Reconocer el error humano como resultado de un fracaso del sistema y no de la persona.

- No castigar a las personas por cometer un error, si no que cuestionar que origino el error y que medidas se pueden adoptar para evitar que se cometan en el futuro.

- Honestidad del personal al momento de realizar un error reportándolo a sus superiores y a los familiares del paciente.

- Tener sistemas informáticos que permitan fomentar y facilitar el reporte de errores e incidentes que son cometidos.

- Implicar a todos los niveles de profesionales en el desarrollo de soluciones que eviten la aparición de errores.

- Aplicar tiempo y recursos de seguridad.

La educación tiene un papel clave en la generación del cambio cultural. En los últimos años, muchos países y organizaciones han gastado una increíble cantidad de trabajo en la preparación de directrices que eduquen y formen en materia de seguridad.

La Alianza Mundial para la Seguridad de los pacientes de la OMS creada el año 2004, ha lanzado la campaña" Cirugía Segura Salva Vidas", en la cual han desarrollado una lista de verificación de seguridad quirúrgica, que apunta a incrementar la seguridad de los pacientes, por medio de asegurar un estándar mínimo básico de seguridad $^{12}$.

Otro aspecto muy importante y poco considerado por el personal de las instituciones hospitalarias es la honestidad frente a los familiares del paciente cuando ocurre un evento adverso, generando un impacto en las acciones legales, financiamiento y los beneficios en la imagen de la institución de salud. El Colegio Americano de Médicos indica que los errores no necesariamente constituyen una conducta indebida, negligente o poco ético, sin embargo, la no revelación de estos si constituyen una conducta indebida. Un estudio realizado frente a 227 casos de incidentes indica los principales motivos por los cuales los pacientes o los familiares decidieron tomar acciones legales ${ }^{12}$ (Tabla 1). El estudio demues- tra que gran cantidad de las acciones legales tomadas fueron para evitar que estos incidentes no vuelvan a ocurrir a otros pacientes, además la necesidad de una explicación juega un rol importante y no debe ser ignorado por las instituciones de salud.

En caso de ocurrir un incidente, existen protocolos confeccionados a nivel mundial, que indican los pasos a seguir para llevar de mejor forma el trato hacia los familiares, los cuales incluyen:

- Continuar los servicios de cuidado.

- Dar a conocer el incidente y las consecuencias generadas en el paciente.

- Entregar información sobre que paso y que esperar frente a la situación.

- Disculpas (cuando sea necesario) o una expresión de arrepentimiento.

- Aviso de tratamientos necesarios.

- Información sobre los cambios y los esfuerzos a realizar para minimizar el impacto.

- Entregar apoyo físico, psicológico, social y financiero.

Las prestaciones ofrecidas a nivel de salud son realizadas en distintas áreas, debido a esto es necesario considerar que la complejidad de las

Tabla 1. Razones por la que se toman las acciones legales

\begin{tabular}{|lc|}
\hline Razones & Porcentaje \\
Para que no pasara nuevamente & 91,4 \\
Necesidad de una explicación & 90,7 \\
$\begin{array}{l}\text { Forzar a que el médico entendiera el error que } \\
\text { cometió }\end{array}$ & 90,4 \\
Para lograr una admisión de negligencia & 86,7 \\
Para que el médico entendiera el sufrimiento & 68,4 \\
causado & \\
Sentimientos ignorados & 66,8 \\
Compensación financiera & 65,6 \\
Enojo & 65,4 \\
Para que el médico no se saliera con la suya & 54,7 \\
Para que el médico fuera disciplinado & 47,6 \\
\hline $\begin{array}{l}\text { Por ser la única forma de expresar los sen- } \\
\text { timientos }\end{array}$ & 45,8 \\
La mala actitud del personal médico & 42,5 \\
\hline Para castigar al médico responsable & 23,2 \\
\hline
\end{tabular}

Tomado de: Best Practice \& Research Clinical Anaesthesiology, H. Van Aken, 2011. 
distintas prestaciones no será igual y se necesitaran distintas medidas de seguridad que permitan evitar posibles daños generados en el paciente, para las distintas áreas del prestador institucional de salud.

Entre las distintas áreas que posee un hospital, el área de pabellón es una de las más criticas, debido a que posee un importante capital y mano de obra especializada, una alta complejidad tecnológica de equipos y sistemas, gran concentración de riesgos, alto nivel de conflictos y una gran dependencia e interdependencia con otros servicios.

La OMS estima que en el mundo alrededor de 234 millones de cirugías son realizadas al año. Estudios realizados en países industrializados han determinado que los pacientes que se someten a una cirugía poseen un porcentaje de mortalidad perioperatoria entre 0,4 a $0,8 \%$ y entre 3 a $17 \%$ de sufrir complicaciones mayores, dichos porcentajes aumentan para el caso de países en vías de desarrollo, por otro lado, la mitad de los eventos adversos que ocurren dentro de un hospital se producen en pabellones.

Las complicaciones quirúrgicas producen un impacto económico no menor que se encuentra entre 12 a $15 \%$ de los costos de atención hospitalaria, esto sugiere que cada hospital debe tener una buena gestión de seguridad a nivel quirúrgico que evite la ocurrencia de incidentes generando a su vez una reducción en los costos.

Se ha identificado que existen distintos factores que son causantes de los incidentes, tales como:

- Poca preocupación en la fase pre-operatoria y post-operatoria: En la fase pre-operatoria mala evaluación del paciente y un manejo inadecuado contribuye entre 38 a $42 \%$ de las muertes $^{13}$. En la fase post-operatoria el riesgo es mayor para los pacientes, la gestión deficiente de la perdida de sangre, supervisión insuficiente del personal de atención o procedimientos inadecuados de reanimación contribuyen con el $43 \%$ de las muertes.

- Errores humanos: Contribuyen con el 51 a $77 \%$ de las muertes ${ }^{13}$. Estos errores se deben principalmente a una falta de experiencia o competencia y a errores de juicio o análisis.

- Trabajo en equipo y comunicación: Un mal trabajo en equipo y una mala comunicación de las distintas personas que componen un equipo de trabajo contribuye con el $62 \%$ de las muertes, una mala comunicación oral contribuye con el $36 \%$ de las muertes y una mala comunicación escrita contribuye con el $20 \%{ }^{13}$. Uno de los aspectos donde se ha observado una mayor debilidad es en el traspaso de los pacientes, debido a cambios de turnos, descansos médicos, transferencias de unidades médicas, derivación y descarga. Un estudio realizado por Borowitz ha identificado que el $31 \%$ del personal medico indicó que durante su turno ocurrió un evento inesperado, debido a que el personal del turno anterior no entrego la información suficiente ${ }^{14}$, otro estudio indica que la primera causa de incidentes en el traspaso es la entrega incompleta de información con un 45,2\% y la no entrega en absoluto de información con un 29,3\% ${ }^{15}$.

- Organización y gestión: Una mala organización y gestión a nivel de inconcordancia de los recursos necesarios, programación quirúrgica, presión de la producción y la inapropiada guardia nocturna contribuyen con el $26 \%$ de las muertes ${ }^{13}$.

Todos los quirófanos de distintos hospitales, están expuestos ha cometer errores los cuales pueden generar diferentes tipos de incidentes o eventos adversos, sin embargo, es de suma importancia que cada hospital confeccione y aplique de forma correcta un programa de seguridad quirúrgica, que considere:

- Mejora en la comunicación y el trabajo en equipo entre los diferentes integrantes del equipo tales como anestesiólogos, cirujanos, enfermeras y arsenaleros, para garantizar que el proceso se esta realizando de la manera correcta. Para lograr un buen funcionamiento, es necesario crear grupos de médicos y enfermeras que confíen entre ellos y sean capaces de discutir, comunicarse y ver claramente que trabajan por un propósito común, escuchar a los pacientes y ser consciente de lo que dicen ellos, de esta forma se pueden obtener resultados que beneficien tanto al medico como al paciente, y por último admitir los errores y comunicarlos tanto a los superiores como a los familiares del paciente afectado.

- Toma de medidas seguridad a nivel pre-operatorio, intra-operatorio y post-operatorio, tanto para el paciente como para el personal médico. 
- Reducir las complicaciones de los procedimientos realizados anticipando e identificando los problemas que podrían generarse a través de simulaciones.

- Reducir al mínimo los errores humanos mediante el uso sistemático de guías clínicas y protocolos.

- Reducir las fallas organizativas mediante la adaptación e implementación de sistemas de gestión desarrollados en otras áreas industriales.

- Prevención de infecciones.

- Registrar el número de procedimientos y resultados de estos en términos de morbilidad, mortalidad, tasa de infecciones e índice de riesgo.

Obtener un nivel de seguridad quirúrgica es un trabajo complejo, debido a la cantidad de aspectos que deben ser considerados, sin embargo, durante años los anestesiólogos se han encargado y han puesto su atención en este tema estudiando a fondo los diferentes aspectos.

Una de las más importantes acciones para generar medidas que mejoren la seguridad a nivel de pabellón es tener un conocimiento de los principales incidentes o eventos adversos a los que esta expuesto el paciente. El aplicar check list de seguridad quirúrgica no implica un tiempo mayor a 3 minutos y permite una importante reducción de las complicaciones y muertes quirúrgicas independientes del entorno económico del país ${ }^{14}$.

Los principales factores causantes de errores en anestesia fueron identificados por CooperJB, el año $1978^{15}$ (Tabla 2), este trabajo concluye que las deficiencias pueden estar presentes en cualquiera de los factores implicados en anestesia, paciente, anestesiólogo, cirujano, enfermeras, auxiliares, máquinas, monitores, fármacos y los relacionados con el ambiente (organización,condiciones de trabajo).

El hombre está expuesto a cometer errores, existen cambios de técnicas, tecnología, personal lo cual predispone a la aparición de errores no previstos, frente a esto es nuestra obligación buscar formas de trabajo seguras, conocer, identificar y detectar la fragilidad del sistema, con la finalidad de prevenir y otorgar una atención de segura de nuestros pacientes.
No olvidemos que la anestesiología moderna persigue dos grandes objetivos seguridad y confort de los pacientes, es importante crear conciencia de Cultura de Seguridad a diferentes niveles de las instituciones de Salud, estos cambios reales los lograremos con Educación e inversión en Seguridad.

"PRIMUN NON NOCERE" Hippocrates 460 a.C.

Tabla 2. Principales factores causantes de errores en anestesia

\begin{tabular}{|l|}
\hline Inexperiencia \\
No estar familiarizado con el equipo o material utilizado \\
Fallas comunicación \\
Precipitación - prisas \\
Falta de atención y de cuidado \\
\hline Distracciones \\
Fatiga \\
\hline Dependencia excesiva de otro personal \\
No realizar el chequeo normal \\
Entrenamiento en el procedimiento \\
Falta de supervisión a médicos y enfermeras en formación \\
Problemas con el entorno,con otros colegas u otros factores \\
Problemas de visión de campo del paciente o equipo (oscu- \\
ridad) \\
\hline Estado físico o mental no adecuado \\
Falta de familiaridad con el procedimiento quirúrgico \\
Mal etiquetado o marcado de las jeringas con fármacos \\
Falta de comprobación \\
Situaciones que imposibilitan las normales precauciones \\
No estar familiarizado con la técnica anestésica \\
Actividad docente en curso \\
Temor o aprensión \\
Caso urgente, gran emergencia \\
Caso difícil \\
Aburrimiento \\
Falta de preparación o planificación \\
Procedimiento muy lento \\
\hline Coopr JB, Newow \\
\hline
\end{tabular}

Cooper JB, Newbower RS, Long CD, McPeek B. Anesthesio$\operatorname{logy} 1978 ; 49$ : 399-406. 


\section{Referencias}

1. Saltman R, Durán A, Dubois H. "The evolving role of hospitals and recent concepts of public sector governance," in Governing Public Hospitals, R. Saltman, A. Durán, and H. Dubois, Eds. 2011, pp. 15-30.

2. Staender S. Incident reporting in anaesthesiology. Best Practice \& Research Clinical Anaesthesiology, H. Van Aken; 2011. pp. 207-14.

3. Whitaker DK, Brattebo G, Smith AF, Staender SE. The Helsinki Declaration on Patient Safety in Anaesthesiology: Putting words into practice. Best Practice \& Research Clinical Anaesthesiology, H. Van Aken; 2011. pp. 277 90.

4. Ortiz A, Alarcón C. Estudio de incidencia de eventos adversos Hospital P. Alberto Hurtado; 2008.

5. Ley Deberes y Derechos del Paciente 20.584 [online], 2012 Diponible en: http://www.leychile.cl//Navegar?idLey $=20584$
6. Sistema de acreditación [online], 2001 Diponible en: http://www. supersalud.gob.cl/568/w3-article-7176.html

7. Arbonés E. L trillo, E. Vilà, "Seguridad y calidad anestésica de la embarazada. Seguridad Del Paciente Quirúrgico, J. Castaño, J. Castillo, F. Escolano, Ll. Gallart, A. Montes, E. Samsó; 2010. pp. 105-15.

8. C. Aibar, “¿Qué es la seguridad del paciente?." in Curso virtual de introducción a la investigación en seguridad del paciente OMS, 2012.

9. Committee on quality of health care in America. Institute of Medicine. To err is human: building a safer health system. Washington: National Academy Press; 1999.

10. Baker GR, Norton PG, Flintoft V, Blais R, Brown A, Cox J, et al. The Canadian Adverse Events Study: the incidence of adverse events among hospital patients in Canada. CMAJ 2004 May;170(11):1678-86.

11. Manser T. Managing the af- termath of critical incidents: Meeting the needs of health-care providers and patients. Best Practice \& Research Clinical Anaesthesiology, H. Van Aken; 2011. pp. 169-79.

12. Haller G, Laroche T, Clergue F. Morbidity in anaesthesia: Today and Tomorrow. Best Practice \& Research Clinical Anaesthesiology, H. Van Aken; 2011. pp. 12331.

13. Borowitz SM, WaggonerFountain LA, Bass EJ, Sledd RM. Adequacy of information transferred at resident sign-out (in-hospital handover of care): a prospective survey. Qual Saf Health Care 2008 Feb;17(1):6-10.

14. Pezzolesi C, Schifano F, Pickles J, Randell W, Hussain Z, Muir $\mathrm{H}$, et al. Clinical handover incident reporting in one UK general hospital. Int J Qual Health Care 2010 Oct;22(5):396-401.

15. Cooper JB, Newbower RS, Long CD, McPeek B. Preventable anesthesia mishaps: a study of human factors. Anesthesiology 1978 Dec;49(6):399-406. 